\title{
The extended Goldman bracket determines intersection numbers for surfaces and orbifolds
}

\author{
MOIRA CHAS \\ SIDDHARTHA GADGIL
}

In the mid eighties Goldman proved that an embedded closed curve could be isotoped to not intersect a given closed geodesic if and only if their Lie bracket (as defined in that work) vanished. Goldman asked for a topological proof and about extensions of the conclusion to curves with self-intersection. Turaev, in the late eighties, asked about characterizing simple closed curves algebraically, in terms of the same Lie structure. We show how the Goldman bracket answers these questions for all finite type surfaces. In fact we count self-intersection numbers and mutual intersection numbers for all finite type orientable orbifolds in terms of a new Lie bracket operation, extending Goldman's. The arguments are purely topological, or based on elementary ideas from hyperbolic geometry.

These results are intended to be used to recognize hyperbolic and Seifert vertices and the gluing graph in the geometrization of three-manifolds. The recognition is based on the structure of the string topology bracket of three-manifolds.

$57 \mathrm{M} 50$

Dedicated with deep and grateful admiration to Bill Thurston (1946-2012)

\section{Introduction}

Goldman [10] discovered in the eighties an intriguing Lie algebra structure on the free abelian group generated by the set of free homotopy classes of closed directed curves on an oriented surface $F$. The definition of the Goldman bracket combines intersection structure with the usual based loop product in the following way: given two closed free homotopy classes $a$ and $b$ with representatives $A$ and $B$ respectively, intersecting only in transversal double points,

$$
[a, b]=\sum_{P \in A \cap B} \operatorname{sign}(P) \widetilde{A \cdot P B},
$$

where $\operatorname{sign}(p)$ is the sign of the intersection between the curves $A$ and $B$ at $P, A \cdot{ }_{p} B$ is the loop product of $A$ and $B$ both viewed as based at $P$, and $\widetilde{C}$ is the free homotopy 
class of a curve $C$. This bracket is extended by linearity to the free module generated by free homotopy classes of curves. Goldman showed that this bracket is well-defined, skew-symmetric and satisfies the Jacobi identity.

Clearly, if $a$ and $b$ are free homotopy classes that have disjoint representatives, then $[a, b]$ is zero. Goldman [10] also showed (using Thurston's earthquakes) that this bracket has the remarkable property that if one of the classes, $a$ or $b$, has a simple representative, then the bracket $[a, b]$ vanishes if and only if $a$ and $b$ can be represented by disjoint curves. Goldman asked for a topological proof and about extensions of the conclusion to curves with self-intersection. Turaev, in the late eighties, asked about characterizing simple closed curves algebraically in terms of this Lie structure.

Later on Chas [7] proved that if either $a$ or $b$ has a simple representative then the bracket of $a$ and $b$ counts the geometric intersection number between $a$ and $b$ (by geometric intersection number we mean the minimum number of points, counted with multiplicity, in which representatives of $a$ and $b$ intersect).

On the other hand, there are examples of classes $a$ and $b$ with no disjoint representatives and such that $[a, b]=0$; see for instance [6, Example 9.1]. The bracket is a homotopy invariant like the set of conjugacy classes in the fundamental group which is, in some sense, simpler than the fundamental group itself. Since intersection and self-intersection numbers of closed curves on surfaces play such a critical role in several areas of lowdimensional topology, it is highly desirable to find characterizations of the intersection numbers. A result of this nature, obtained by Chas and Krongold [8], was that for the subset of compact orientable surfaces with non-empty boundary, the bracket $\left[a, a^{3}\right]$ determines the self-intersection number of $a$.

Finally, after twenty five years since Goldman's paper [10] we show here how the bracket answers the question about disjunction and simplicity of closed curves for all finite type surfaces. We also count self-intersection numbers and mutual intersection numbers for all finite type orientable orbifolds in terms of a new Lie bracket operation, extending Goldman's. Our results fill in most of the lacunae in partial results that have resisted extension over the intervening years. The arguments are purely topological, using group theory ideas of Freedman, Scott and Hass [17; 9], or they are based on elementary geometrical ideas from hyperbolic geometry.

By a Fuchsian group we mean a discrete group of orientation-preserving isometries of the hyperbolic plane. Below are the two main results of this paper.

Mutual Intersection Theorem Let $x$ and $y$ be non-conjugate hyperbolic elements in a finitely generated Fuchsian group. Consider the generalized Goldman bracket $[\cdot, \cdot]$ of the $p^{\text {th }}$ power of $x$ with the $q^{\text {th }}$ power of $y$, where $p$ and $q$ are such that the 
ratio $p / q$ is different from the ratio of the translation length of $x$ and the translation length of $y$. Then for all but finitely many values of $p$ and $q$ (which are explicit from the proof), the geometric intersection number of $x$ and $y$ is given by the number of terms in $\left[\left\langle x^{p}\right\rangle,\left\langle y^{q}\right\rangle\right]$, counted with multiplicity, divided by $p \cdot q$.

Self-Intersection Theorem For $x$ a hyperbolic element in a finitely generated Fuchsian group, which is not a proper power of another element, the geometric selfintersection number of $x$ is given by the number of terms (counted with multiplicity) divided by $p \cdot q$ of $\left[\left\langle x^{p}\right\rangle,\left\langle x^{q}\right\rangle\right]$, for all but finitely many pairs of distinct positive integers $p$ and $q$. (Once more, the excluded pairs are determined explicitly by the proof.)

Our proof is based on the word hyperbolicity of Fuchsian groups rather than smallcancellation theory as in [8]. By extending the result of [8] for surfaces with boundary to closed surfaces we complete the answer to Goldman's question [10, Subsection 5.17] as to whether his topological result (that if $a$ and $b$ are two free homotopy classes of curves on a surface such that $a$ has a simple representative and $[a, b]=0$, then $a$ and $b$ have disjoint representatives) has a topological proof.

The main lemma of this work states that if at least one of $p$ and $q$ is sufficiently large and the lengths of $x^{p}$ and $y^{q}$ are different, then there is no cancellation of terms in the bracket $\left[\left\langle x^{p}\right\rangle,\left\langle y^{q}\right\rangle\right]$. In other words, if the representatives $A$ and $B$ intersect in the minimum number of points, then two intersection points $P$ and $Q$ with different sign do not give the same free homotopy class of curves, that is, $\overline{A \cdot P B} \neq \widehat{A \cdot Q B}$.

We show this by constructing quasigeodesic representatives of a lift of a loop representing $A \cdot_{P} B$. These quasigeodesics are the concatenations of certain segments of translates of the axis of $x$ and the axis of $y$. As quasigeodesics are not too far from geodesics, it follows that if two points of intersection give the same free homotopy class, then there is a pair of corresponding quasigeodesics that are close, which then implies that they are equal. We deduce that the two points correspond to terms with the same sign in the Goldman bracket.

For the final step (deducing that two points correspond to terms with the same sign), rather than using general $\delta$-hyperbolicity arguments as sketched above, we use hyperbolic geometry and the fact that the quasigeodesic curves we construct are actually piecewise geodesic and are explicitly described. This gives a sharper result than one would get with general arguments: for our result, we only require that one of the exponents $p$ and $q$ is large, while coarser geometric arguments would require both to be large. 
These results are intended to be applied to recognize hyperbolic and Seifert vertices and the gluing graph in the geometrization of three-manifolds. The recognition is based on the structure of the string topology bracket of three-manifolds.

For a typical irreducible three-manifold, the cyclic homology of the group ring of the fundamental group lives in two degrees: zero and one. Degree one is a Lie algebra and degree zero is a Lie module for degree one. The Lie algebra breaks into a direct sum corresponding to the pieces and the module structure tells how they are combined in the graph.

One can show that the Goldman bracket on the linear space with basis the set of free homotopy classes and the power operations on this basis determine the Fuchsian group of an orbifold. Thus, the Goldman bracket solves the "recognition problem" for twodimensional orbifolds. More significantly, now that the proof of the Geometrization conjecture has enabled a classification of three-manifolds, there arises the need to calculate the geometrization in examples like knots, ie the "recognition problem for three-manifolds". Our work directly applies to that since the string topology bracket in three-manifolds will be used to describe the canonical graph of the geometrization picture as well as which vertices are hyperbolic and which are Seifert fibered spaces. This bracket is largely concentrated on the Seifert pieces. On these pieces it depends on the orbifold bracket defined here. The orbifold part of the story seemed sufficiently interesting to present independently with the details of the application to three-manifolds coming next.

We emphasize though that the above characterization is a new one for closed curves on closed surfaces, and should be of interest even in this case.

Others have considered string topology operations for orbifolds and manifold stacks in a more abstract setting, see for instance Ángel, Backelin and Uribe [1], Behrend, Ginot, Noohi and $\mathrm{Xu}$ [3], and Lupercio, Uribe and Xicotencatl [15]. It would be interesting to relate those results to the concrete results here.

Acknowledgements This paper benefited from conversations with Ian Agol, Danny Calegari and Dennis Sullivan. It started when the first author was visiting the Indian Institute of Science in Bangalore, India, to which she would like to express gratitude. An insightful and diligent referee is responsible for many improvements. Finally, in the final stage of this paper, the authors learned the sad news of Bill Thurston's death. This work wouldn't have been possible without the many directions he opened up in mathematics.

Supported by NSF grant 1071448-1-46676. 
Outline In Section 2 we review the group theoretic definition of intersection number from [9] and [17] as well as the definition of the geometric intersection number of closed curves in a two-dimensional, orientable orbifold. Section 3 is devoted to the extension of the Goldman bracket to oriented orbifolds (a crucial part of this definition is the elementary geometric fact that if two hyperbolic transformations $x$ and $y$ have intersecting axes, then $x \cdot y$ is hyperbolic). In Section 4 we prove the Jacobi identity for the extension of the Goldman bracket (interestingly enough, this proof boils down to the proposition of geometry that if a line intersects a side of a triangle, then it intersects one of the other two sides). In Section 5 we give examples of the bracket in the modular surface (a beautiful and computable example of orbifolds). In Section 6 we show that geodesics are quantitatively separated for hyperbolic surfaces (and orbifolds): namely if two closed geodesics are sufficiently close and parallel after lifting to the universal cover, they must coincide. In Section 7 we prove the main non-cancellation lemma, stating that if the conjugacy classes of the two terms of the bracket coincide, then the two quasigeodesics associated to these two terms coincide. Finally in Section 8 we give the proofs of the intersection theorem and the self-intersection theorem.

\section{The geometric intersection number and the group theoretic intersection number}

Let $G$ be a discrete subgroup of orientation-preserving isometries of the hyperbolic plane $\mathbb{H}$. (The set of isometries of $\mathbb{H}$, Isom $(\mathbb{H})$ has the compact-open topology.)

Each isometry $g$ of the hyperbolic plane extends to the circle at infinity, where, if $g \neq 1$, it fixes at most 2 points. An isometry is called elliptic, parabolic or hyperbolic according as it fixes 0,1 or 2 points respectively in the circle at infinity. A hyperbolic isometry $g$ fixes the (hyperbolic) line joining its two fixed points at infinity. This line is called the axis of $g$. Further, the sets of fixed points at infinity of two isometries contained in a discrete subgroup $G$ are either disjoint or coincide. If the sets of fixed points at infinity of a pair of elements of $G$ coincide and are non-empty, then the isometries are both powers of the same element of $G$.

In this paper, an orbifold $\mathbb{H} / G$ is the quotient of the hyperbolic plane $\mathbb{H}$ by a discrete group of orientation-preserving isometries $G$, provided with the induced metric. The pertinent finer notion of free homotopy for orbifolds is described in Section 2.1. (Note that we are using the word "orbifold" in a narrower sense than the usual.)

In this section we review the definition of closed curves, homotopy and geometric intersection number for curves for an orbifold (Section 2.1), the group theoretic definition of intersection number in orbifolds (Section 2.2), and show these two definitions agree. 
(The reader is referred to [18, Chapter 13], [4, Chapter 2] and [13, Section 6.2] for a more general definition of orbifolds and orbifold homotopy. See also [16, Section 13.3] for a formidable discussion of based orbifold homotopy in terms of charts.)

\subsection{Orbifold homotopy and the geometric intersection number}

A cone point $P$ in $\mathbb{H} / G$ is the projection of a point in $\mathbb{H}$ which is fixed by some non-trivial element of $G$. The order of a cone point $P$ is the cardinality of the maximal cyclic subgroup of $G$ fixing $P$.

Consider the projection map, $\Pi: \mathbb{H} \rightarrow \mathbb{H} / G$. A representative of a closed oriented curve in an orbifold $\mathbb{H} / G$ is a continuous map $\alpha: \mathbb{S}^{1} \rightarrow \mathbb{H} / G$ (with $\mathbb{H} / G$ thought of as a topological space), passing through at most finitely many cone points, together with the choice of a full lift $\hat{\alpha}: \mathbb{R} \rightarrow \mathbb{H}$, so that $\Pi \circ \hat{\alpha}=\alpha \circ \Theta$, where $\Theta: \mathbb{R} \rightarrow \mathbb{R} / 2 \pi \mathbb{Z}$ is the usual projection. Two representatives of closed curves are equivalent if their full lifts are related by an element of the group $G$. A closed curve on the orbifold $\mathbb{H} / G$ is an equivalence class of representatives of closed curves.

Definition 2.1 Two closed oriented curves $\alpha$ and $\alpha^{\prime}$ in $\mathbb{H} / G$ are $\mathbb{H} / G$-homotopic if they are related by a finite sequence of moves. Each of these moves is either a homotopy in the complement of the cone points or is one of the skein relations or moves depicted in Figures 1 and 2. There, the disk where the move happens contains exactly one cone point $P$, and $n$ denotes the order of $P$. An arc with no self-intersection in the disk and passing through $P$ is $\mathbb{H} / G$-homotopic relative to endpoints to an arc spiraling around $P$ in either direction ( $n-1) / 2$ times if $n$ is odd (Figure 2), or $n / 2$ times if $n$ is even (Figure 1). Also, if $n$ is odd, the endpoints of the arc are antipodal and if $n$ is even, the endpoints coincide.

Remark 2.2 The skein relations depicted in Figures 1 and 2 imply that a loop going $n$ times in either direction around a point of order $n$ can be "erased" from a closed curve (Figure 3). However, note that the skein relation in Figure 3 is less precise than Definition 2.1. Namely, this relation does not "tell" as Definition 2.1 does tell how to homotope a curve passing through a cone point. Since some geodesics do pass through cone points, we need the skein relation in Definition 2.1 that deals with those cases.

The proof of the next result is very similar to that of the (standard) proof of a bijection between free (usual) homotopy classes of closed curves on a path-connected space and conjugacy classes of the fundamental group of the space (see, for instance, [12, Chapter 1, Exercise 6]).

Theorem 2.3 There is a natural bijection between the set of conjugacy classes of $G$ and the set of $\mathbb{H} / G$-free homotopy classes of closed oriented curves in $\mathbb{H} / G$. 
$\mathbb{H}$
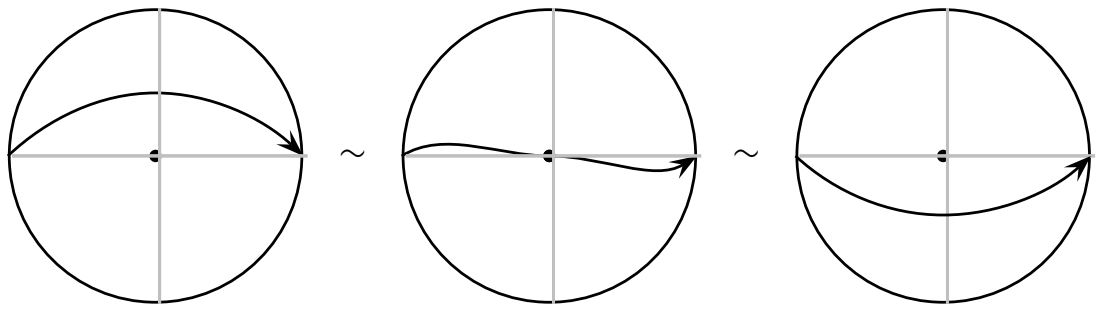

$\mathbb{H} / G$
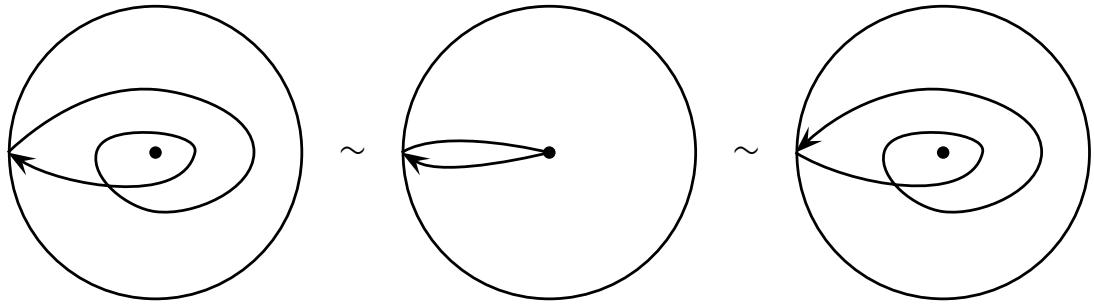

Figure 1: Skein relations for points of order $n=4$ (bottom) and the corresponding lifts (top)

$\mathbb{H}$
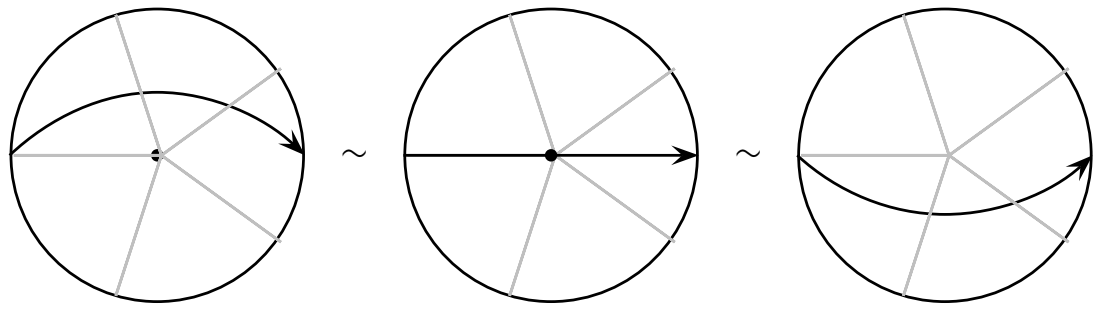

$\mathbb{H} / G$
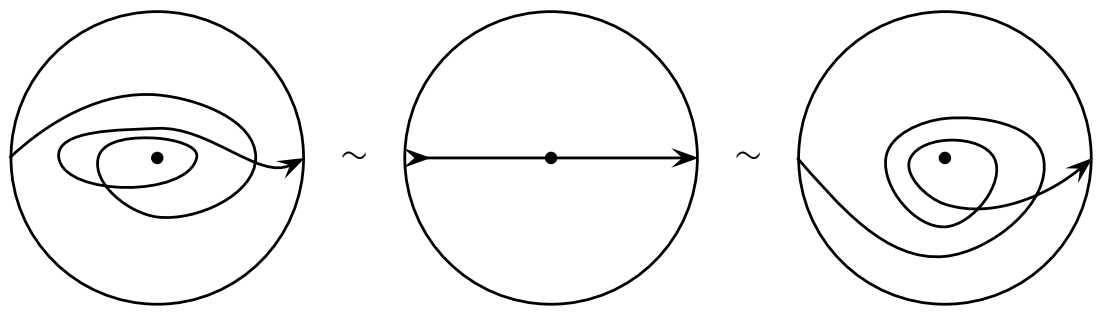

Figure 2: Skein relations for points of order $n=5$ (bottom) and the corresponding lifts (top)

If $a$ and $b$ in are two elements of $\mathbb{H} / G$, the intersection number of $a$ and $b$ is the minimum number (counted with multiplicity) of transversal intersection points of pairs of loops representing $a$ and $b$ not passing through cone points.

Remark 2.4 If at least one of the elements, $a$ or $b$, belongs to the conjugacy class of a non-hyperbolic element of $G$ then the intersection number of $a$ and $b$ is zero. 
Case $n=2$
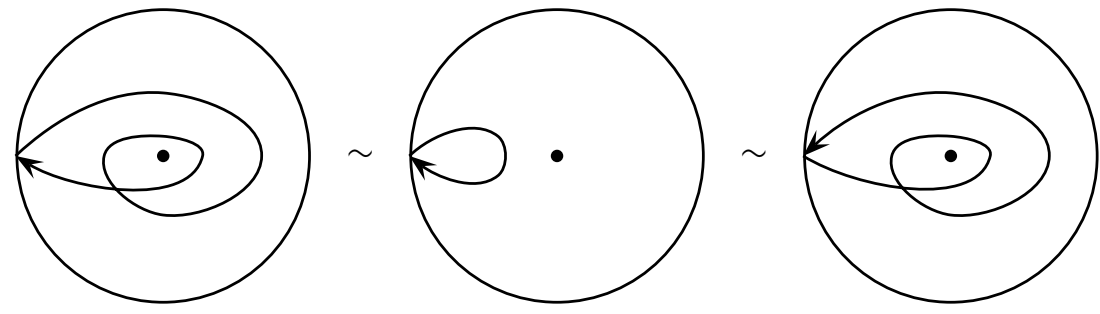

Case $n=3$
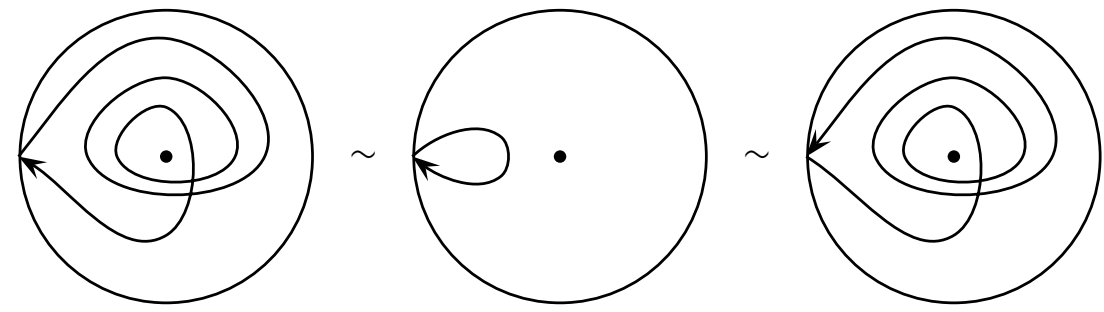

Figure 3: Consequence of skein relations for points of order $n=2$ (top) and $n=3$ (bottom)

(Conjugacy classes of elements of $G$ are identified with free homotopy classes of curves on $\mathbb{H} / G$ via Theorem 2.3.)

\subsection{Labeling intersection points: the group theoretic intersection number}

A hyperbolic isometry $x$ acts on its axis $A_{x}$ by translation by a real number $\tau_{x}$, the translation length of $x$. We orient the axis $A_{x}$ so that for each point $P$ in $A_{x}$, the direction from $P$ to $x P$ is positive.

Let $x, y \in G$. Denote by $X \backslash G / Y$ the space of double cosets $X g Y$ where $g \in G$, and $X$ and $Y$ denote the cyclic subgroups generated by $x$ and $y$ respectively. If $x$ or $y$ is not hyperbolic, set $I(x, y)=\varnothing$, otherwise, set

$$
I(x, y)=\left\{X g Y \in X \backslash G / Y \text { such that } A_{x} \cap g A_{y} \neq \varnothing\right\} .
$$

Scott [17] discusses intersection numbers of closed curves on compact surfaces. The next proposition can be proven by arguments completely analogous to those of Scott [17, Section 1]. The point is that $\mathbb{H} / G$-homotopy after lifting becomes exactly like usual homotopy in the universal cover. Thus our discussion and Scott's are the same, mutatis mutandis, as far as the proposition below is concerned. (In the next proposition, the identification of conjugacy classes in $G$ and $\mathbb{H} / G$-free homotopy classes of closed curves in $\mathbb{H} / G$ given by Theorem 2.3 is used.)

Proposition 2.5 Let $x$ and $y$ be elements of $G$. Then the intersection number of the conjugacy classes of $x$ and $y$ equals the cardinality of $I(x, y)$. 


\section{The Goldman bracket for orbifolds}

Recall that $\mathcal{C}$ denotes the set of conjugacy classes of elements in $G$. Consider $\mathbb{Z}[\mathcal{C}]$, the free module generated by $\mathcal{C}$. For $x \in G$, let $\langle x\rangle$ denote the conjugacy class of $x$. In particular, $\langle x\rangle \in \mathbb{Z}[\mathcal{C}]$.

In this section we will define a linear map $[\cdot, \cdot]: \mathbb{Z}[\mathcal{C}] \otimes \mathbb{Z}[\mathcal{C}] \rightarrow \mathbb{Z}[\mathcal{C}]$ and show in Section 4 that it is a Lie bracket. This bracket generalizes Goldman's to orientable two-dimensional orbifolds and will be defined (as Goldman's) on two elements of the basis of $\mathbb{Z}[\mathcal{C}]$ by considering the intersection points of a certain pair of representatives (see Section 2.2), assigning a signed free homotopy class to each of these points (the signed product at the intersection point) and adding up all those terms.

For elements $a$ and $x$ in $G$, let $x^{a}$ denote $a x a^{-1}$. If $x$ is hyperbolic, the isometry $x^{a}$ is also hyperbolic, has the same translation length as $x$, ie $\tau_{x^{a}}=\tau_{x}$, and the axis of $x^{a}$ is given by $a \cdot A_{x}$. From now on, fix an orientation of $\mathbb{H}$. Also, for $x$ and $y$ in $G$ set $\iota(x, y)$ to be zero if $x$ or $y$ are elliptic or parabolic or if the axes of $x$ and $y$ do not cross, and to be the sign of the crossing, otherwise. Finally, set

$$
[\langle x\rangle,\langle y\rangle]=\sum_{X b Y \in I(x, y)} \iota\left(x, y^{b}\right)\left\langle x y^{b}\right\rangle .
$$

Notation 3.1 Let $P$ be a point in the axis $A_{x}$ of a hyperbolic transformation $x$. If $r$ is a positive real number, $S(x, P, r)$ denotes the segment of $A_{x}$ of length $r$ starting (and including) $P$, but not the other endpoint, in the positive direction of $A_{x}$. If $r$ is a negative number, $S(x, P, r)$ denotes the segment of $A_{x}$ starting at a point $Q$ at distance $r$ from $P$ in the negative direction, containing $Q$ but not $P$.

Remark 3.2 Fix a point $P$ in $A_{x}$ and let $r$ be the translation length of $x$. Let

$$
J(x, y, P)=\left\{g Y \in G / Y: S(x, P, r) \cap g A_{y} \neq \varnothing\right\} .
$$

Then there is a bijection between $I(x, y)$ and $J(x, y, P)$. Since $G$ is a discrete group, both sets have finite cardinality. Moreover,

$$
[\langle x\rangle,\langle y\rangle]=\sum_{g Y \in J(x, y, P)} \iota\left(x, y^{g}\right)\left\langle x y^{g}\right\rangle .
$$

Remark 3.3 The conjugacy classes of elliptic and parabolic elements of $G$ are in the center of the Lie algebra; that is, the bracket between these classes and all other classes is zero. 
Remark 3.4 By [2, Theorem 7.38.6], if $x$ and $y$ are hyperbolic isometries whose axes intersect then $x y$ is also hyperbolic. Moreover, the axis of $x y$ and its translation length can be determined as follows (see [2] for details). Denote by $P$ the intersection point of $A_{x}$ and $A_{y}$. Denote by $Q$ the point on $A_{x}$ at distance $\tau_{x} / 2$ from $P$ in the positive direction of $A_{x}$ and by $R$ the point on $A_{y}$ at distance $\tau_{y} / 2$ from $P$ in the negative direction of $A_{y}$. The axis of $A_{x y}$ is the oriented line from $R$ to $Q$ and the translation length of $x y$ equals twice the distance between $R$ and $Q$. (See Figure 4; this is one of the "triangles" mentioned in the introduction which are used to unravel the Jacobi relation.)

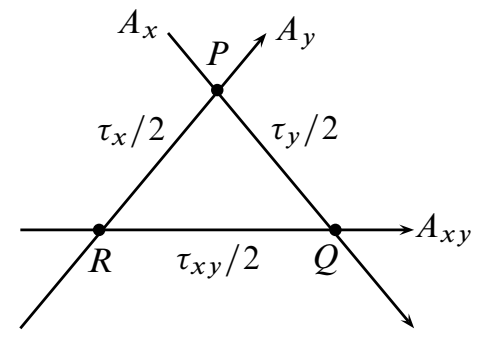

Figure 4: The axis of $x y$

Remark 3.5 Consider the set of pairs of cosets $G / X \times G / Y$. The group $G$ acts on the set $G / X \times G / Y$ by $(g X, h Y) \mapsto(a g X, a h Y)$, for each $a \in G$. Denote by $D(x, y)$ the quotient under this action. Set $f: D(x, y) \rightarrow X \backslash G / Y$ by mapping the equivalence class of $(g X, h Y)$ to $X g^{-1} h Y$. A straightforward computation shows that $f$ is well defined and it is a bijection. Also, the preimage under $f$ of an element $X k Y$ of $I(x, y)$ is the set of equivalence classes of pairs of cosets $(g X, h Y)$ such that $g A_{x} \cap h A_{y} \neq \varnothing$ and $g^{-1} h=k$. Moreover,

$$
[\langle x\rangle,\langle y\rangle]=\sum_{(a X, b Y) \in D(x, y)} \iota\left(x^{a}, y^{b}\right)\left\langle x^{a} y^{b}\right\rangle .
$$

\section{Triple brackets and the Jacobi identity}

The Jacobi identity for the extended bracket can probably be proved by arguments analogous to those used by Goldman in his proof that the bracket of curves on surfaces satisfies it.

In this section we present a geometric proof of the Jacobi identity, that does not use transversality.

Let $x$ be a hyperbolic isometry. The next result is stated using Notation 3.1. 
Lemma 4.1 The following equation holds (see Figure 5):

$$
\begin{aligned}
{[[\langle x\rangle,\langle y\rangle],\langle z\rangle]=} & \sum_{(X g Y, X h Z) \in T} \iota\left(x, y^{g}\right) \iota\left(x, z^{h}\right)\left\langle x y^{g} z^{h}\right\rangle \\
& +\sum_{(X g Y, Y h Z) \in U} \iota\left(x, y^{g}\right) \iota\left(y^{g}, z^{h}\right)\left\langle x y^{g} z^{h}\right\rangle,
\end{aligned}
$$

where

$$
\begin{array}{r}
T=\left\{(X g Y, X h Z): \text { for some } P \in A_{x}, A_{x} \cap g A_{y}=\{P\}, S\left(x, P, \tau_{x}\right) \cap h A_{z} \neq \varnothing,\right. \\
h A_{z} \cap\left(S\left(y^{g}, P,-\tau_{y} / 2\right) \cup S\left(y^{x g}, x P, \tau_{y} / 2\right)=\varnothing\right\}, \\
U=\left\{(X g Y, Y h Z): \text { for some } P \in A_{x}, A_{x} \cap g A_{y}=\{P\},\right. \\
\left(S\left(y^{g}, P,-\tau_{y} / 2\right) \cup S\left(y^{x g}, x P, \tau_{y} / 2\right)\right) \cap h A_{z} \neq \varnothing, \\
\left.S\left(x, P, \tau_{x}\right) \cap h A_{z}=\varnothing\right\} .
\end{array}
$$

Proof Let $g \in G$ such that $A_{x} \cap g A_{y} \neq \varnothing$. We can retrace the steps of the construction described in Remark 3.4 to find $A_{x y^{g}}$ (Figure 5). Next, we compute $\left[\left\langle x y^{g}\right\rangle,\langle z\rangle\right]$. Denote by $P$ the intersection point between $A_{x}$ and $g A_{y}$, by $S$ the intersection point of $A_{x}$ with $A_{x y^{g}}$ and by $R$ the intersection point of $g A_{y}$ and $A_{x y}$. Finally, denote by $Z$ the cyclic group generated by $z$. By Remark 3.2,

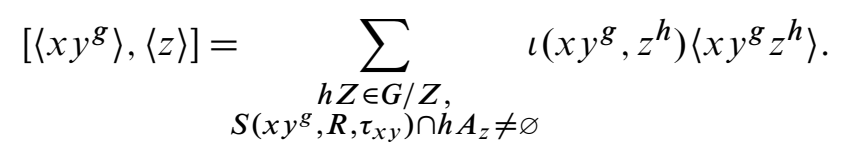

Let $h Z \in G / Z$. Observe that the inequality $S\left(x y^{g}, R, \tau_{x y}\right) \cap h A_{z} \neq \varnothing$ holds if and only if $h A_{z}$ crosses either the triangle with vertices $R, P, S$ or the triangle with vertices $S, x P, x y^{g} R$ (Figure 5). Thus, $h A_{z}$ intersects $S\left(x y^{g}, R, \tau_{x y}\right)$ if and only if exactly one of the following holds:

(1) $S\left(x, P, \tau_{x}\right) \cap h A_{z} \neq \varnothing$ and $\left(S\left(y^{g}, P,-\tau_{y} / 2\right) \cup S\left(y^{x g}, x P, \tau_{y} / 2\right)\right) \cap h A_{z}=\varnothing$, or

(2) $S\left(x, P, \tau_{x}\right) \cap h A_{z}=\varnothing$ and $\left(S\left(y^{g}, P,-\tau_{y} / 2\right) \cup S\left(y^{x g}, x P, \tau_{y} / 2\right) \cap h A_{z} \neq \varnothing\right.$.

The first pair of conditions corresponds to a term in the first sum, and the second pair of conditions corresponds to terms in the second sum.

This concludes the proof.

A corollary is the Jacobi identity. 


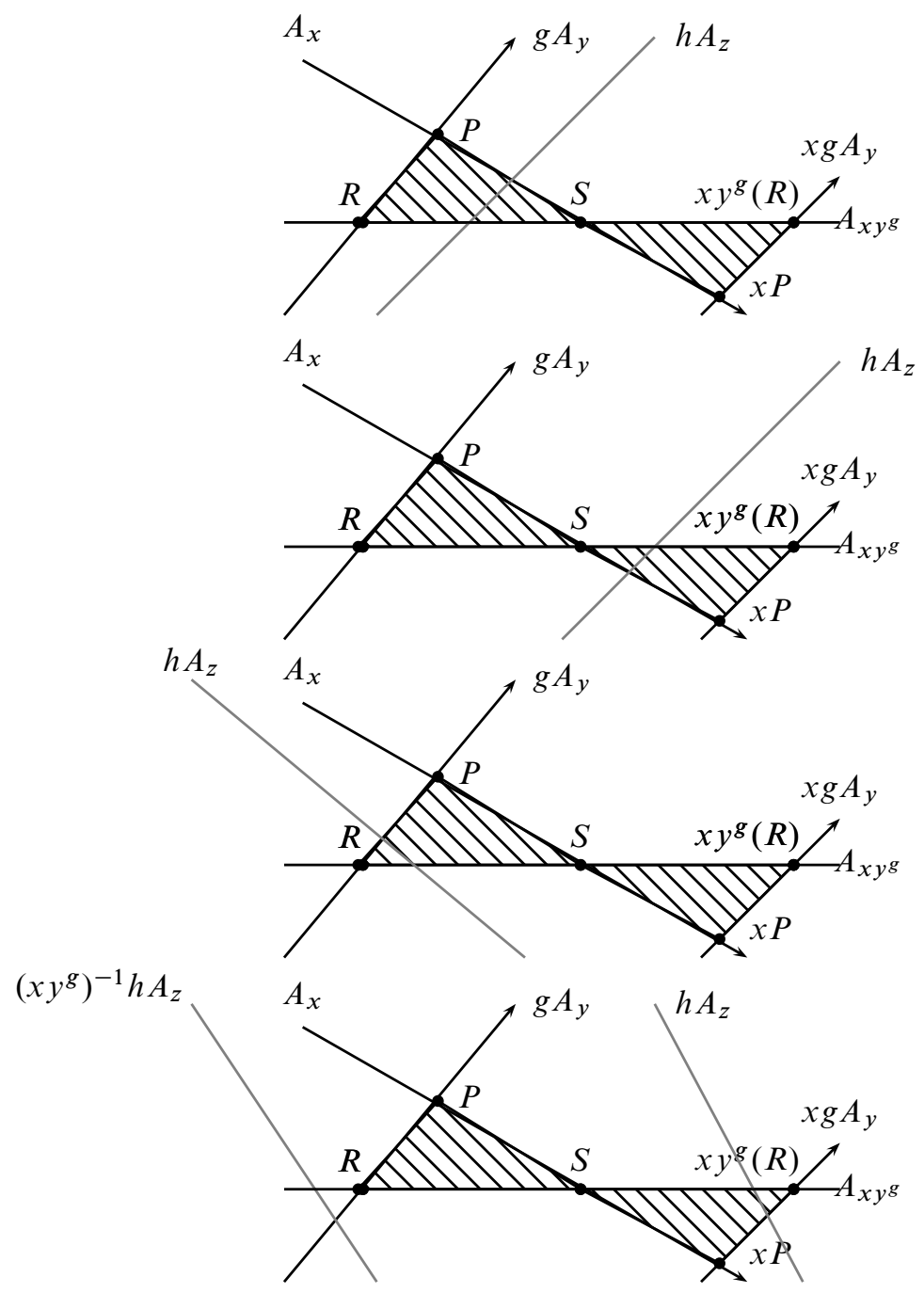

Figure 5: Jacobi identity

Theorem 4.2 For $x, y, z \in G$,

$$
[[\langle x\rangle,\langle y\rangle],\langle z\rangle]+[[\langle y\rangle,\langle z\rangle],\langle x\rangle]+[[\langle z\rangle,\langle x\rangle],\langle y\rangle]=0 .
$$

Therefore, $[\cdot, \cdot]: \mathbb{Z}[\mathcal{C}] \otimes \mathbb{Z}[\mathcal{C}] \rightarrow \mathbb{Z}[\mathcal{C}]$ is a Lie bracket.

Proof The three terms of the Jacobi relation after applying Lemma 4.1 decompose into six groups of terms. Among these, the pairs corresponding to the triangles of Figure 5 cancel. 


\section{Examples}

Consider the modular group $\operatorname{PSL}(2, \mathbb{Z})$, that is, the group consisting of all transformations $z \rightarrow(a z+b) /(c z+d)$, where $a, b, c, d \in \mathbb{Z}$ and $a d-b c=1$. This group is generated by $T(z)=z+1$ and $S(z)=-1 / z$, with relations $S^{2}=1$ and $(S T)^{3}=1$. The modular group is a finitely generated, discrete subgroup of orientation-preserving isometries of the hyperbolic plane. Therefore, the bracket can be defined on the free module generated by conjugacy classes.

Orient the hyperbolic plane clockwise.

By computing the traces, one can see that the elements $x=T S T T$ and $y=T T T S T T T$ of $\operatorname{PSL}(2, \mathbb{Z})$ are hyperbolic and not conjugate.

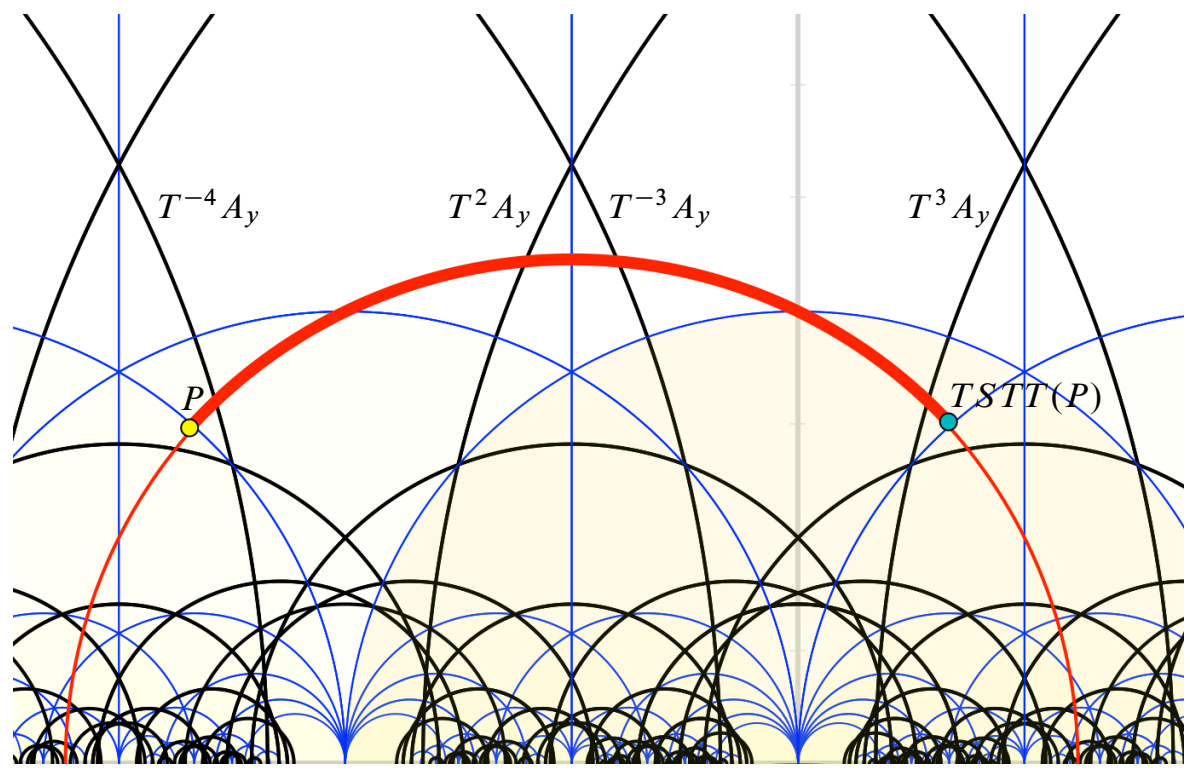

Figure 6: Translates of $A_{y}$ (in black), and a fundamental domain of $A_{x}$ (in thick red) where $x=T S T T$ and $y=T T T S T T T$

As shown in Figure 6 , there are exactly four translates of $y$ by $\operatorname{PSL}(2, \mathbb{Z})$ that intersect the segment of $A_{x}$ from the point $P$ to $\operatorname{TTST}(P)$.

In this example, $I(x, y)=\left\{X T^{-4} Y, X T^{-3} Y, X T^{2} Y, X T^{3} Y\right\}$. The term corresponding to the double coset $X T^{-4} Y$ has positive sign and is the conjugacy class of $S T^{6}$ because $x T^{-4} y T^{4}=T S T S T^{7}=S T^{6}$. The term corresponding to $X T^{3} Y$ has negative sign and is the conjugacy class of $x T^{3} y T^{-3}=T S T^{8} S$. This element is conjugate to $S T S T^{8}=T^{-1} S T^{-1} T^{8}$. Thus the term corresponding to the double 
coset $X T^{3} Y$ is $-\left\langle S T^{6}\right\rangle$ Also, the terms associated to $X T^{-3} Y$ and $X T^{2} Y$ are $+\left\langle S T T S T^{7}\right\rangle$ and $-\left\langle S T T S T^{7}\right\rangle$. Thus $[\langle x\rangle,\langle y\rangle]=0$.

In order to study the brackets of $\left\langle x^{p}\right\rangle$ and $\left\langle y^{q}\right\rangle$ when $p$ and $q$ are larger than one, one can use the criteria given in [14] for conjugacy in $\operatorname{SL}(2, \mathbb{Z})$ (and therefore in $\operatorname{PSL}(2, \mathbb{Z}))$. Doing so, one can check that $\left[\langle x\rangle,\left\langle y^{3}\right\rangle\right] \neq 0$. Moreover, the number of terms of the bracket $\left[\langle x\rangle,\left\langle y^{3}\right\rangle\right]$ (counted with multiplicity) equals twelve, which is three times the intersection number of $\langle x\rangle$ and $\langle y\rangle$.

In the same way one can see that $\left[\langle x\rangle,\left\langle x^{2}\right\rangle\right]=0$ and $\left[\langle x\rangle,\left\langle x^{3}\right\rangle\right]$ has 24 terms, which is six times the self-intersection number of $\langle x\rangle$.

The above calculations are computer-assisted: one looks at Figure 6 (done with Cinderella) to identify the terms, then uses Mathematica to calculate the terms, and study cancellation.

\section{Quantitative separation of geodesics}

From now on, we assume that the discrete subgroup $G$ of $\operatorname{Isom}(\mathbb{H})$ is finitely generated.

Definition 6.1 Fix $\delta>0$, two geodesics $\Gamma$ and $\Gamma^{\prime}$ and two (not necessarily distinct) points $P$ and $Q$ in $\Gamma$ and $\Gamma^{\prime}$ respectively. We say that $\Gamma$ and $\Gamma^{\prime}$ are $\delta$-close at $P$ and $Q$ if $d(P, Q)<\delta$ and, if $\Upsilon$ denotes a geodesic passing through $P$ and $Q$, then the absolute value of the difference between the corresponding angles between $\Upsilon$ and $A_{x}$ and between $\Upsilon$ and $A_{y}$ (in the positive direction of both axes) is less than $\delta$. If there exist points $P$ and $Q$ such that two geodesics $\Gamma$ and $\Gamma^{\prime}$ are $\delta$-close at $P$ and $Q$, then we say that $\Gamma$ and $\Gamma^{\prime}$ are $\delta$-close.

The next lemma is well known to experts but we include a proof here because we were unable to find one in the literature.

Lemma 6.2 For each $L>0$ there exists a $\delta>0$ such that if $x$ and $y$ are two hyperbolic transformations in $G$ such that $\tau_{x} \leq L$ and $\tau_{y} \leq L$ and $A_{x}$ and $A_{y}$ are $\delta$-close, then $A_{x}=A_{y}$.

Proof Denote by $\lambda$ the hyperbolic convex hull of the limit set of $G$. (Recall that the limit set of $G$ is the set of accumulation points of any $G$-orbit in $\mathbb{H}$.) Since $G$ is finitely generated, by [11, Lemma 1.3.1 and Theorem 1.3.2], there exists a subset $\lambda^{*}$ of $\lambda$, invariant under $G$, such that the quotient of $\lambda^{*}$ by $G$ is compact and the axis of every hyperbolic transformation in $G$ intersects $\lambda^{*}$. Thus, there exists a compact, convex subset $C$ of $\mathbb{H}$ such that $\lambda^{*} \subset G \cdot C$. 
Fix a positive number $L$ and denote by $C^{\prime}$ the closure of the $(L+1)$-neighborhood of $C$.

Claim 1 Given $\varepsilon>0$ there exists a $\delta>0$ such that if $x$ and $y$ are hyperbolic transformations whose axes are $\delta$-close and whose transformation lengths are bounded above by $L$, then $d(R,[x, y] R)<\varepsilon$ for all $R \in C^{\prime}$.

We argue by contradiction. Suppose that there exist $\varepsilon>0$ and two sequences $\left\{x_{n}\right\}$ and $\left\{y_{n}\right\}$ of hyperbolic transformations with translation length bounded above by $L$ and such that for each $n, x_{n}$ and $y_{n}$ are $1 / n$-close, $A_{x_{n}} \neq A_{y_{n}}$ and there exists a point $R_{n} \in C^{\prime}$ that satisfies $d\left(R_{n},\left[x_{n}, y_{n}\right] R_{n}\right)>\varepsilon$.

Claim 2 For each $n$, we can assume that the points $P_{n}$ and $Q_{n}$ in $A_{x_{n}}$ and $A_{y_{n}}$ realizing Definition 6.1 are in $C^{\prime}$.

Indeed, denote by $P_{n}^{\prime}$ and $Q_{n}^{\prime}$ the points in $A_{x_{n}}$ and $A_{y_{n}}$ realizing Definition 6.1.

The axis $A_{x_{n}}$ projects to a closed geodesic $a_{n}$ in $\mathbb{H} / G$. Since the translation length of $x_{n}$ is bounded above by $L$, so is the length of $a_{n}$. On the other hand, $A_{x_{n}}$ intersects $G \cdot C$. Hence, the projection of $P_{n}^{\prime}$ to $\mathbb{H} / G$ is at distance at most $L$ from the projection of $G \cdot C$. Thus there is an element $g \in G$ such that $g P_{n}^{\prime}$ is at distance at most $L$ from $C$. Since $Q_{n}^{\prime}$ is close to $P_{n}^{\prime}$, we have that $Q_{n}^{\prime}$ is also in $C^{\prime}$. The proof of Claim 2 is completed by replacing the sequences $\left\{x_{n}\right\}$ and $\left\{y_{n}\right\}$ by the sequences $\left\{g x_{n} g^{-1}\right\}$ and $\left\{g y_{n} g^{-1}\right\}$.

Claim 3 The sequences $\left\{x_{n}\right\}$ and $\left\{y_{n}\right\}$ have subsequences converging to hyperbolic transformations $x$ and $y$ respectively.

Consider the sequences $\left\{T_{n}\right\}$ and $\left\{S_{n}\right\}$ of endpoints of $\left\{A_{x_{n}}\right\}$ in the circle at infinity in the negative and positive directions respectively. Since the circle is compact, by taking subsequences, we can assume that $\left\{T_{n}\right\}$ and $\left\{S_{n}\right\}$ converge to $T$ and $S$ respectively. Since each $A_{x_{n}}$ intersects the compact set $C^{\prime}$, we get $T \neq S$. Analogously, the sequence $\left\{\tau_{x_{n}}\right\}$ of translation lengths is bounded above by $L$. Therefore, it has a convergent subsequence. Thus, Claim 3 follows.

Since $A_{x_{n}}$ and $A_{y_{n}}$ are $1 / n$-close, we get $A_{x}=A_{y}$. Hence, $[x, y] P=P$ for all $P \in \mathbb{H}$. On the other hand, by taking a convergent subsequence of $\left\{R_{n}\right\}$, we see that $d(R,[x, y] R) \geq \varepsilon$ for some $R \in C^{\prime}$. This contradiction completes the proof of Claim 1 .

To finish the proof of the lemma, observe that since $G$ is discrete, there exists an open subset $U$ of isometries of $\mathbb{H}$ such that the identity is the only element of $G$ in $U$. Let

$$
V_{\eta}=\left\{g \in \operatorname{PSL}(2, \mathbb{R}) \mid d(R, g R)<\eta \text { for all } R \text { in } C^{\prime}\right\} .
$$


There exists an $\varepsilon>0$ such that $V_{\varepsilon} \subset U$. On the other hand, by Claim 1, there exists a $\delta>0$ such that if the axes of $x$ and $y$ are $\delta$-close, then $[x, y] \in V_{\varepsilon}$. Thus, the bracket $[x, y]$ equals the identity, which implies $A_{x}=A_{y}$.

Corollary 6.3 For each $L>0$ and each $C>0$ there exists a constant $M>0$ such that for every pair of hyperbolic elements $x$ and $y$ in $G$ with different axes and such that $\tau_{x}<L$ and $\tau_{y}<L$, the set $A_{x} \cap N_{C}\left(A_{y}\right)$ is a (possibly empty) geodesic segment of length at most $M$.

Proof Let $\delta$ be as in Lemma 6.2 for $L$ and $G$ and let $N$ be the length of the (possibly empty) segment $A_{x} \cap N_{C}\left(A_{y}\right)$.

If $A_{x}$ and $A_{y}$ intersect at an angle $\theta$, then by Lemma $6.2, \sin (\theta) \geq \sin (\delta)$. By the rule of $\operatorname{sines}, \sinh (N / 2) \leq \sinh (C) / \sin (\delta)$ (see Figure 7, left). Then $N$ is bounded above by a constant depending on $C$ and $\delta$.

If $A_{x}$ and $A_{y}$ are parallel, by Lemma 6.2 they are at distance at least $\delta$. Since the distance between $A_{x}$ and $A_{y}$ is realized, there is a quadrilateral as in Figure 7, right, with all angles except $\theta$ being right angles, $A \geq \delta$ and $B \leq C$.
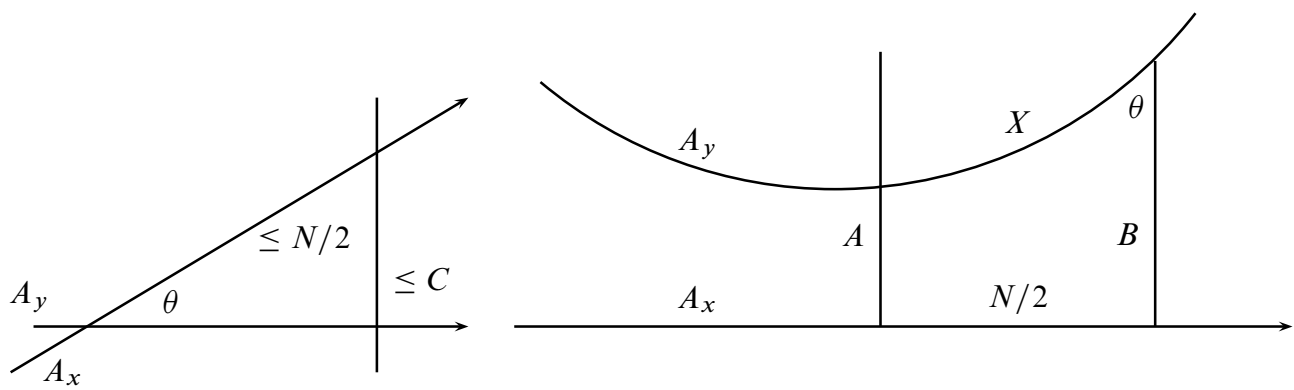

Figure 7: Proof of Corollary 6.3

By [2, Theorem 7.17.1(i)], $\sinh (N / 2)=\cos (\theta) / \sinh (A) \leq 1 / \sinh (\delta)$ (see Figure 7, right). This implies that $\cosh (N / 2)$ is bounded above by a bound depending on $\delta$. An elementary computation gives the desired result.

\section{The non-cancellation lemma}

Let $K$ be a real positive number. A piecewise-smooth embedding $\gamma$ of $\mathbb{R}$ in the hyperbolic plane is a $K$-quasigeodesic if for any pair of points $P$ and $Q$ in $\gamma$, the length of the path in $\gamma$ joining $P$ and $Q$ is at most $K \cdot d(P, Q)$. 
Fix a pair of hyperbolic elements $x$ and $y$ in $G$ whose axes intersect at a point $P$. We will describe the construction of a piecewise-smooth embedding $\gamma$ of $\mathbb{R}$ (depending on $x$ and $y$ ) and show it is a quasigeodesic.

Let $\alpha:[0,1] \rightarrow \mathbb{H}$ be the curve from $\alpha(0)=y^{-1} P$ to $\alpha(1)=x P$, whose image is given by the concatenation of the geodesic segment of $A_{y}$ from $y^{-1} P$ to $P$ with the geodesic segment of $A_{x}$ from $P$ to $x P$. Since $x y(\alpha(0))=\alpha(1), \alpha$ can be extended by periodicity to a map $\gamma(x, y): \mathbb{R} \rightarrow \mathbb{H}$ such that $\gamma(x, y)(t)=\alpha(t)$ for $t \in[0,1]$ and $\gamma(x, y)(t+1)=x y \gamma(x, y)(t)$ for all $t$.

The map $\gamma(x, y)$ is a piecewise geodesic curve consisting of segments of length $\tau_{x}$ (included in the axes of conjugates of $A_{x}$ by some power of $x y$ ) alternating with segments of length $\tau_{y}$ (included in the axes of conjugates of $A_{y}$ by some power of $x y$ ).

We remark that we will be using more than just that $\gamma(x, y)$ is a quasigeodesic, but also its geometric nature. Indeed purely abstract results about quasigeodesics suffice to prove a weaker version of our result, where we need to assume that both $p$ and $q$ are large.

Lemma 7.1 For each $L>0$ there exists a constant $K>0$ depending on $G$ such that if $x$ and $y$ are hyperbolic transformations in $G$ whose axes are distinct and intersect, and whose translation lengths are bounded above by $L$, then for each pair of positive integers $p$ and $q$, the curve $\gamma\left(x^{p}, y^{q}\right)$ is a $K$-quasigeodesic. Moreover, the oriented angles between any pair of consecutive maximal segments of $\gamma\left(x^{p}, y^{q}\right)$ are congruent.

Proof Fix $p$ and $q$ and repeat the construction of Remark 3.4 for the hyperbolic isometries $x^{p}$ and $y^{q}$. The transformation $x^{p}$ maps the angle determined by $y^{-q} P$, $P, x^{p}(P)$ to the angle $x^{p} y^{-q} P, x^{p} P, x^{2 p}(P)$ (Figure 8). Thus, these two angles are congruent. The angle $x^{p} y^{-q} P, x^{p} P, x^{2 p}(P)$ is congruent to the angle $P$, $x^{p} P, x^{p} y^{q}(P)$ because they are opposite at the intersection of $A_{x}$ and $x^{p} y^{q}\left(A_{y}\right)=$ $A_{x^{p} y x^{-p}}$. This implies that the angles determined by $y^{-q} P, P, x^{p}(P)$ and by $P$, $x^{p}(P), y^{q} x^{p}(P)$ are congruent. Therefore the angles formed by the consecutive maximal segments of $\gamma\left(x^{p}, y^{q}\right)$ (labeled with $\theta_{1}$ in Figure 8) are all congruent.

Denote by $T$ the triangle with vertices $y^{-q} P, P, x^{p}(P)$ and by $T^{\prime}$ the triangle with vertices $P, x^{p}(P)$ and $x^{p} y^{q}(P)$, see Figure 8 . Since $T$ and $T^{\prime}$ have an angle and the two adjacent sides to the angle congruent, they are congruent.

Set $g=x^{p} y^{q}$. Then $A_{g}$ is invariant under $g$, so $A_{g}$ crosses the middle of the band $\bigcup_{k \in \mathbb{Z}} g^{k}\left(T \cup T^{\prime}\right)$.

To prove that $\gamma\left(x^{p}, y^{q}\right)$ is a quasigeodesic, observe that triangles

$$
g^{s}(T), g^{s}\left(T^{\prime}\right), g^{s+1}(T), g^{s+1}\left(T^{\prime}\right), \ldots, g(T), g\left(T^{\prime}\right), \ldots, g^{r}(T), g^{r}\left(T^{\prime}\right)
$$




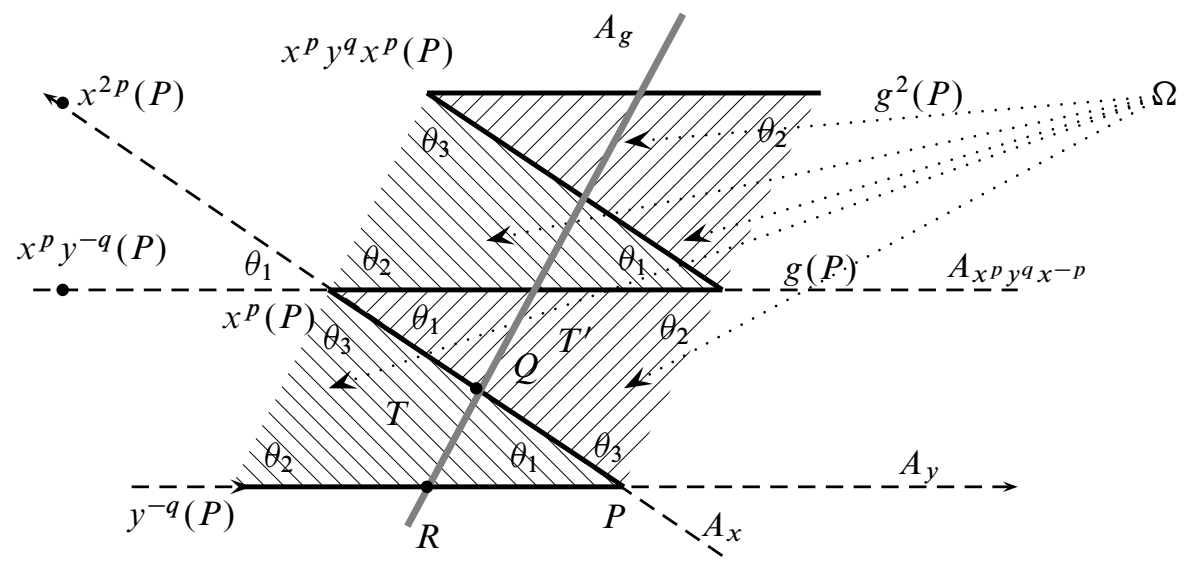

Figure 8: Quasigeodesic associated to $x, y, p$ and $q$ (here $g=x^{p} y^{q}$ )

form a polygon $\Omega$. On the other hand, since the angles $\theta_{1}, \theta_{2}$ and $\theta_{3}$ (see Figure 8) are the interior angles of a triangle, they add up to at most $\pi$. This implies that the polygon $\Omega$ is convex. Therefore, the geodesic between two points in the curve $\gamma$ is in the interior of $\Omega$. By elementary hyperbolic geometry, there exists a positive constant $K$ such that $\gamma$ is a $K$-quasigeodesic. (Note that $K$ can be taken so that it depends only on the lower bound of the angle between intersecting elements of axes of hyperbolic elements in $G$ given by Lemma 6.2.)

We can (and will) assume without loss of generality that $K \geq 1$.

Lemma 7.2 Let $L>0$ and let $K>0$ be the constant of Lemma 7.1. Then there exists a constant $C>0$ depending on $G$ such that if $x$ and $y$ are hyperbolic transformations in $G$ whose axes are distinct and intersect, and whose translation lengths are bounded above by $L$, then for each pair of positive integers $p$ and $q$, the $K$-quasigeodesic $\gamma\left(x^{p}, y^{q}\right)$ satisfies $\gamma\left(x^{p}, y^{q}\right) \subset N_{C / 2}\left(A_{g}\right)$ and $A_{g} \subset N_{C / 2}\left(\gamma\left(x^{p}, y^{q}\right)\right)$, where $g=x^{p} y^{q}$.

Proof Denote by $d[p, q]$ the distance between $P$ (the point in $A_{x} \cap A_{y}$ ) and $A_{g}$. Consider the region $\lambda$ bounded by the axes $A_{x}$ and $A_{y}$ and the arc of the circle of center $P$ and radius $d[p, q]$. The area of $\lambda$ equals $2 \theta_{1} \sinh ^{2}(d[p, q] / 2)$. Also, $\lambda$ is included in the triangle $T$, of area bounded above by $\pi-\theta_{1}$ (see Figure 9). Hence,

$$
2 \sinh ^{2}(d[p, q] / 2) \leq\left(\pi-\theta_{1}\right) / \theta_{1} \leq \pi / \delta .
$$

Therefore, there exists a constant $C_{1}>0$ such that $d[p, q] \leq C_{1}$ for all positive integers $p$ and $q$. Observe (Figure 8) the distance between any point in $\gamma\left(x^{p}, y^{q}\right)$ and $A_{g}$ is smaller than $d[p, q]$. This implies $\gamma\left(x^{p}, y^{q}\right) \subset N_{C_{1}}\left(A_{g}\right)$. 


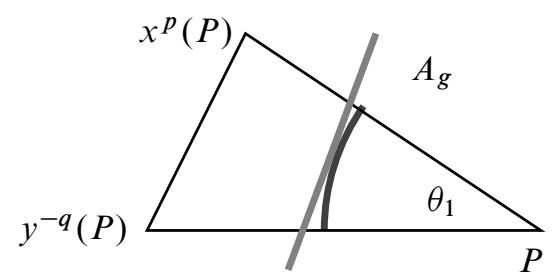

Figure 9: The region $\lambda$

Denote by $R$ the intersection point of $A_{g}$ with $A_{x}$ and by $Q$ the intersection point of $A_{g}$ with $A_{y}$ (see Figure 8).

Consider the triangle with vertices $P, Q$ and $R$. Triangles in the hyperbolic plane $\mathbb{H}^{2}$ are $\ln (1+\sqrt{2})$-thin [5, Fact 4, page 90]. In particular, the side of the triangle included in $A_{g}$ is at distance at most $\ln (1+\sqrt{2})$ from the union of the other two sides.

By taking $C=2 \max \left\{\ln (1+\sqrt{2}), C_{1}\right\}$ the desired result follows.

Let $x$ and $y$ be two hyperbolic transformations in $G$ whose axes intersect at a point $P$ and whose length is less than $L$. Let $p$ and $q$ be positive integers. Denote by $I$ the segment of $A_{x}$ from $P$ to $x^{p}(P)$.

For a subsegment $J$ of $I$ with endpoints $S$ and $R$, we consider a rectangular neighborhood $U=U(J, C)$ defined as follows. Let $s$ (resp. $r$ ) be the open half-plane bounded by the line perpendicular to $A_{x}$ through $S$ (resp. $R$ ), containing the point $x^{p} P$ (resp. $P$ ). Set $U=s \cap r \cap N_{C}(I)$.

Note that the boundary of $U$ consists of vertical segments contained in the boundaries of $s$ and $r$ and horizontal segments contained in the boundary of $N_{C}(I)$. By elementary hyperbolic geometry, the distance between the vertical segments is the length of the geodesic $J$.

Lemma 7.3 Let $L>0$ and let $x$ and $y$ be two hyperbolic transformations in $G$ whose axes intersect at a point $P$ and whose length is less than $L$. Let $p$ and $q$ be positive integers such that $p \cdot \tau_{x} \geq 6 K C$, where $K$ and $C$ are as in Lemmas 7.1 and 7.2. Denote by $I$ the segment of $A_{x}$ from $P$ to $x^{p}(P)$.

Let $S$ and $R$ be the points in $A_{x}$ at distance $3 K C$ from $P$ and $x^{p} P$, and let $J$ be the segment from $S$ to $R$. Let $U=U(J, C)$ be the associated rectangular neighborhood. Then closure $(U) \cap N_{C}(\lambda)=\varnothing$ for all maximal geodesic segments of $\lambda$ of $\gamma\left(x^{p}, y^{q}\right)$ distinct from $I$. 
Proof Let $Q \in \lambda$, where $\lambda$ is a maximal segment of $\gamma\left(x^{p}, y^{q}\right)$ different from $I$, and let $T \in I$ be a point. Then by construction the length of a path in $\gamma\left(x^{p}, y^{q}\right)$ from $Q$ to $T$ is at least $3 K C$. As $\gamma\left(x^{p}, y^{q}\right)$ is a $K$-quasigeodesic, it follows that $d(Q, T)>3 C$. As $Q$ was an arbitrary point of $\lambda$ and $U \subset N_{C}(\lambda)$ it follows that $\operatorname{closure}(U) \cap N_{C}(L)=\varnothing$.

Observe that $U$ contains the open subsegment $J$ of length at least $p \cdot \tau_{x}-6 K C$.

The following lemma is key to the paper.

Lemma 7.4 For each $L>0$ there exists a positive integer $p_{0}$ such that for each pair of integers $p$ and $q$ satisfying $p \geq p_{0}$, and for each pair of hyperbolic transformations $x, y$ and $x_{1}, y_{1}$ whose axes are distinct and intersect, and whose translation length is bounded above by $L$, if $x^{p} y^{q}=x_{1}^{p} y_{1}^{q}, x_{1}$ is conjugate to $x$, and $y_{1}$ is conjugate to $y$, then $\gamma\left(x^{p}, y^{q}\right)=\gamma\left(x_{1}^{p}, y_{1}^{q}\right)$.

Proof We start by informally describing the two parts of the proof. First, in the situation above, the two corresponding quasigeodesics are such that one is in a $C$-neighborhood of the other. In particular, segments of one quasigeodesic are in $C$-neighborhoods of segments of the other quasigeodesic. By making the integer $p$ large enough, we obtain a "long" geodesic segment in a $C$-neighborhood of other geodesic segment. This implies that these two segments intersect in an interval.

Second, we use the fact that the quasigeodesics are constructed by translating two consecutive maximal segments by powers of $g$, to show if the two intersecting segments are distinct, an impossible figure is obtained.

Here are the details of the proof. For each finitely generated, discrete subgroup $G$ of $\operatorname{Isom}(\mathbb{H})$, there exists a positive constant $\tau_{0}$ such that for each hyperbolic transformation $x \in G$, one has $\tau_{x} \geq \tau_{0}$ (see, for instance, [11, Theorem 1.4.2])

Let $C$ and $K$ be as in Lemmas 7.1 and 7.2. Let $M$ be the constant of Corollary 6.3. We will show that $p_{0}=K(3 M+6 C) / \tau_{0}$ gives the desired conclusion.

Since $x^{p} y^{q}=x_{1}^{p} y_{1}^{q}$, we have $A_{x^{p} y^{q}}=A_{x_{1}^{p}} y_{1}^{q}$. By Lemma 7.2,

$$
\gamma\left(x_{1}^{p}, y_{1}^{q}\right) \subset N_{C / 2}\left(A_{g}\right) \subset N_{C}\left(\gamma\left(x^{p}, y^{q}\right)\right) .
$$

Let $U$ and $J$ respectively be the neighborhood and the segment given by Lemma 7.3, so $J \subset U, J \subset I \subset \gamma\left(x^{p}, y^{q}\right)$ and the length of $J$ is at least $p \tau_{x}-6 K C$.

Observe that $\gamma\left(x_{1}^{p}, y_{1}^{q}\right)$ must intersect $U$, for otherwise $\gamma\left(x_{1}^{p}, y_{1}^{q}\right)$ is included in $N_{C}\left(\gamma\left(x^{p}, y^{q}\right) \backslash J\right)$, which has two components. Furthermore, $\gamma\left(x_{1}^{p}, y_{1}^{q}\right)$ must intersect both components, contradicting the fact that $\gamma\left(x_{1}^{p}, y_{1}^{q}\right)$ is connected. By 
Lemma 7.3, $N_{C}(L) \cap \operatorname{closure}(U)=\varnothing$ for all maximal segments $\lambda$ of $\gamma\left(x^{p}, y^{q}\right)$ distinct from $I$. Hence, $\gamma\left(x_{1}^{p}, y_{1}^{q}\right)$ does not intersect the horizontal boundary components of $U$, as otherwise we obtain points in $\gamma\left(x_{1}^{p}, y_{1}^{q}\right)$ whose distance from $\gamma\left(x^{p}, y^{q}\right)$ is greater than $C$.

By hypothesis, the length of $J$ is at least $p \tau_{x}-6 K C$ so it is at least $3 K M$.

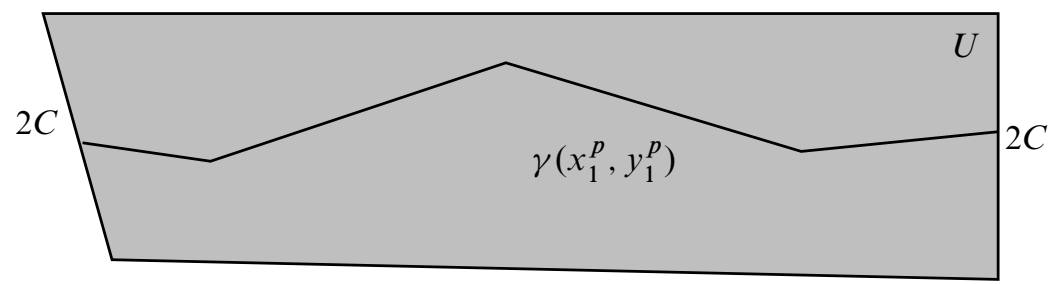

Figure 10: The intersection of neighborhood $U$ of $J$ with $\gamma\left(x_{1}^{p}, y_{1}^{q}\right)$

Thus, the components of the set $U \cap \gamma\left(x_{1}^{p}, y_{1}^{q}\right)$ are piecewise linear curves starting and ending at the vertical sides of $U$ (see Figure 10). Let $\beta$ be one of these components. We claim that $\beta$ contains a segment $l$ of length greater than $M$. Indeed, if $\beta$ contains three or more vertices of $\gamma\left(x_{1}^{p}, y_{1}^{q}\right)$ then one segment of $\beta$ is a maximal segment of $\gamma\left(x_{1}^{p}, y_{1}^{q}\right)$ included in a translate of $x_{1}^{p}$. Therefore, it must have length at least $p_{0} \tau_{x}$. Otherwise, $\beta$ consists of at most three segments. Denote by $m$ the length of the longest of these segments. As the distance between the vertical boundary components of $U$ is the length of $J$,

$$
3 K M \leq p \tau_{x}-6 K C \leq 3 m .
$$

Since $K>1, m>M$. Thus the claim is proved.

The segment $l$ of $\beta$ of length at least $M$ is included in some segment $I^{\prime}$ of $\gamma\left(x_{1}^{p}, y_{1}^{q}\right)$. Thus $I^{\prime} \cap N_{C}(J)$ contains a segment longer than $M$. By Corollary 6.3, $I^{\prime}$ intersects $I$ in a subsegment. This concludes the first part of the proof. We will show that the assumption $I \neq I^{\prime}$ leads to a contradiction.

If $I \neq I^{\prime}$, by interchanging the roles of $I$ and $I^{\prime}$ if necessary, we can assume that there is a vertex $v$ of $I$ which is not in $I^{\prime}$. Let $v^{\prime}$ be the vertex of $I^{\prime}$ closest to $v$. Denote by $\lambda$ (resp. $\lambda^{\prime}$ ) the maximal segment of $\gamma\left(x^{p}, y^{q}\right)$ (resp. $\left.\gamma\left(x_{1}^{p}, y_{1}^{q}\right)\right)$ such that $I$ and $\lambda$ (resp. $I^{\prime}$ and $\lambda^{\prime}$ ) are adjacent and intersect in $v$ (resp. $v^{\prime}$ ).

Recall that $\gamma\left(x^{p}, y^{q}\right)$ (resp. $\left.\gamma\left(x_{1}^{p}, y_{1}^{q}\right)\right)$ is constructed by taking two consecutive maximal segments and translating them by powers of $g$. To simplify the notation, we write $g=x^{p} y^{q}$. The segment adjacent to $\lambda$ (resp. $\lambda^{\prime}$ ) different from $I$ (resp. $I^{\prime}$ ) 
is $g(I)$ (resp. $\left.g\left(I^{\prime}\right)\right)$. Denote by $u$ (resp. $\left.u^{\prime}\right)$ the other vertex of $I$ (resp. $\left.I^{\prime}\right)$. Note that $v$ and $g(u)$ (resp. $v^{\prime}$ and $\left.g\left(u^{\prime}\right)\right)$ are the vertices of $\lambda$ (resp. $\lambda^{\prime}$ ).

Suppose first that $u$ is in $I^{\prime}$. By Lemma 7.1, the angles $u, v, g(u)$ and $v, g(u), g(v)$ are congruent. Hence there is a convex quadrilateral with vertices $v, v^{\prime}, g(u), g\left(u^{\prime}\right)$, see Figure 11. By Lemma 7.1, the sum of the interior angles of this quadrilateral is $2 \pi$, a contradiction in hyperbolic geometry. This implies that $u$ is not in $I^{\prime}$.

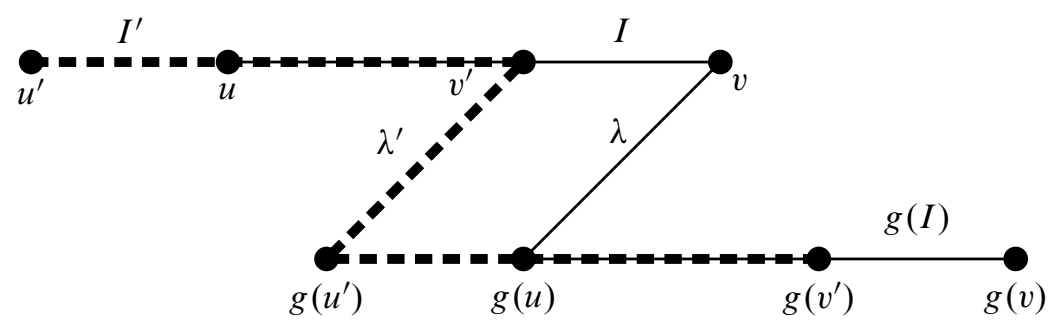

Figure 11: Length of $I$ equals the length of $I^{\prime}$

Denote by $l$ the geodesic through $v$ and $g(u)$. By Lemma 7.1, the angles $u, v$, $g(u)$ and $v, g(u), g(v)$ are congruent. This implies that $u$ and $g(v)$ are in different sides of $l$. On the other hand, $u$ and $v^{\prime}$ (resp. $g(v)$ and $g\left(u^{\prime}\right)$ ) are on the same side of $l$. Then $v^{\prime}$ and $g\left(u^{\prime}\right)$ are on different sides of $l$. Hence $\lambda$ intersects $\lambda^{\prime}$ and the quasigeodesics are arranged as in Figure 12.

In particular, the segments $\lambda$ and $\lambda^{\prime}$ intersect at a point $z$. The triangles with vertices $z, v^{\prime}, v$ and $z, g(u), g\left(u^{\prime}\right)$ have congruent corresponding angles. Hence, these two triangles are congruent. Thus, $z$ is the middle point of $\lambda$, and also of $\lambda^{\prime}$. Since the segments with vertices $u, u^{\prime}$ and $g(u), g\left(u^{\prime}\right)$ are congruent, the segments with vertices $u, u^{\prime}$ and $v^{\prime}, v$ are congruent.

Denote by $w$ the middle point of $I$. Observe that $w$ is also the middle point of $I^{\prime}$ (as segments with vertices $u, u^{\prime}$ and $v, v^{\prime}$ are congruent). As $x_{1}$ is conjugate to $x$ and $y_{1}$ is conjugate to $y$, the length of the arc of $\gamma\left(x_{1}^{p}, y_{1}^{q}\right)$ from $w$ to $z$ equals $\left(p \tau_{x}+q \tau_{y}\right) / 2$. Also, the length of the arc of $\gamma\left(x^{p}, y^{q}\right)$ from $w$ to $z$ equals $\left(p \tau_{x}+q \tau_{y}\right) / 2$. By the triangle inequality, this is impossible. Thus we conclude that $v=v^{\prime}$, and hence also $u=u^{\prime}$.

Thus, we see that $I=I^{\prime}$ and $\lambda=\lambda^{\prime}$. It follows that the quasigeodesics $\gamma\left(x^{p}, y^{q}\right)$ and $\gamma\left(x_{1}^{p}, y_{1}^{q}\right)$ coincide as they are the unions of translates under $g$ of $I \cup \lambda$ and $I^{\prime} \cup \lambda^{\prime}$, respectively. 


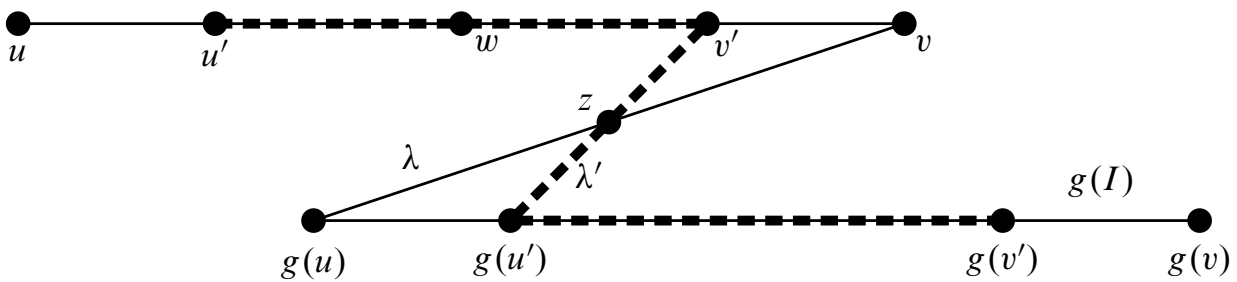

Figure 12: Length of $I$ is larger than length of $I^{\prime}$

Theorem 7.5 For each $L>0$ there exists a positive integer $p_{0}$ such that for each pair $p \geq p_{0}$ and $q$ of positive integers, if $x$ and $y$ (resp. $x_{1}$ and $y_{1}$ ) are hyperbolic transformations whose axes are distinct and intersect, $x$ is conjugate to $x_{1}, y$ is conjugate to $y_{1}$, the translation lengths of $x, x_{1}, y, y_{1}$ are bounded above by $L$, $p \tau_{x} \neq q \tau_{y}$, and $x^{p} y^{q}=x_{1}^{p} y_{1}^{q}$, then there exists an $h \in G$ such that $x_{1}=x^{h}$ and $y_{1}=y^{h}$.

Proof Since $x^{p} y^{q}=x_{1}^{p} y_{1}^{q}$, we have $A_{x^{p}} y^{q}=A_{x_{1}^{p}} y_{1}^{q}$. Moreover, both axes are oriented in the same direction. If $p_{0}$ is the positive integer given by Lemma 7.4, then $\gamma\left(x^{p}, y^{q}\right)=\gamma\left(x_{1}^{p}, y_{1}^{q}\right)$. Hence, by the definition of $\gamma\left(x^{p}, y^{q}\right)$, if $g=x^{p} y^{q}$ there exists an $n \in \mathbb{Z}$ such that one of the following holds:

(1) $x_{1}^{p}=\left(x^{p}\right)^{g^{n}}$ and $y_{1}^{q}=\left(y^{q}\right)^{g^{n}}$.

(2) $x_{1}^{p}=\left(y^{q}\right)^{g^{n+1}}$ and $y_{1}^{q}=\left(x^{p}\right)^{g^{n}}$.

Since $p \tau_{x} \neq q \tau_{y},(2)$ is impossible. Thus the result follows by taking $h=g^{n}$.

\section{Proof of the main theorem}

An element $z$ in $\mathbb{Z}[\mathcal{C}]$ can be uniquely represented as a sum $\sum_{i=1}^{k} n_{i}\left\langle x_{i}\right\rangle$ so that the conjugacy classes $\left\langle x_{i}\right\rangle$ are all distinct and the integers $n_{i}$ are non-zero. We define the Manhattan norm of $z$ by

$$
M\left(\sum_{i=1}^{k} n_{i}\left\langle x_{i}\right\rangle\right)=\sum_{i=1}^{k}\left|n_{i}\right|
$$

We are now in a position to prove our main theorem. Denote by $X_{p}$ and $Y_{q}$ the cyclic groups generated by $x^{p}$ and $y^{q}$ respectively. Note that by definition

$$
\left[\left\langle x^{p}\right\rangle,\left\langle y^{q}\right\rangle\right]=\sum_{X_{p} b Y_{q} \in I\left(x^{p}, y^{q}\right)} \iota\left(x^{p},\left(y^{q}\right)^{b}\right)\left\langle x^{p}\left(y^{q}\right)^{b}\right\rangle .
$$




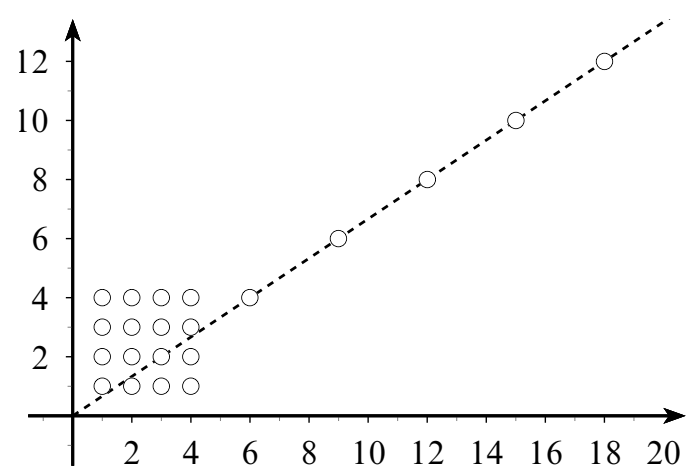

Figure 13: Values of $p$ and $q$ in the main theorem, $p_{0}=5, p / q \neq \frac{3}{2}, p$ in $x$-axis

Our first step is to collate terms in this expression. There is a natural quotient map from $X_{p} \backslash G / Y_{q}$ to $X \backslash G / Y$, mapping $X_{p} \backslash g / Y_{q}$ to $X \backslash g / Y$. Observe that $\iota\left(x^{p},\left(y^{q}\right)^{b}\right)=$ $\iota\left(x, y^{b}\right)$. Further observe that if $X_{p} \backslash g / Y_{q}$ and $X_{p} \backslash g^{\prime} / Y_{q}$ map to the same element in $X g Y$, then $\left\langle x^{p}\left(y^{q}\right)^{g}\right\rangle=\left\langle x^{p}\left(y^{q}\right)^{g^{\prime}}\right\rangle=\left\langle x^{p}\left(y^{g^{\prime}}\right)^{q}\right\rangle$. The lemma below follows by grouping terms corresponding to their images in $I(x, y)$.

Lemma 8.1 We have

$$
\left[\left\langle x^{p}\right\rangle,\left\langle y^{q}\right\rangle\right]=p q\left(\sum_{X b Y \in I(x, y)} \iota\left(x, y^{b}\right)\left\langle x^{p}\left(y^{b}\right)^{q}\right\rangle\right) .
$$

We are now ready to prove our main result.

Main Theorem Let $G$ be a finitely generated, discrete group of $\operatorname{Isom}(\mathbb{H})$ and let $L>0$. There exists a $p_{0}$ such that if $p$ and $q$ are integers at least one of which is larger than $p_{0}$, then the following holds:

(1) If $x$ and $y$ are hyperbolic transformations in $G$ such that neither is conjugate to a power of the other, with translation lengths bounded above by $L$ and such that $p \tau(x) \neq q \tau(y)$, then $M\left[x^{p}, y^{q}\right] /(p \cdot q)$ equals the geometric intersection number of $x$ and $y$.

(2) If $p \neq q$, and $x$ is a hyperbolic transformation in $G$, not a proper power, and has translation length bounded above by $L$, then $M\left[x^{p}, x^{q}\right] /(2 \cdot p \cdot q)$ equals the geometric self-intersection number of $x$.

Proof Interchanging $x$ and $y$ if necessary, we can assume that $p \geq p_{0}$.

Suppose that $\left\langle x^{p}\left(y^{b}\right)^{q}\right\rangle=\left\langle x^{p}\left(y^{b^{\prime}}\right)^{q}\right\rangle$. Then for some $h \in G$,

$$
x^{p}\left(y^{b}\right)^{q}=\left(x^{p}\left(y^{b^{\prime}}\right)^{q}\right)^{h}=\left(x^{p}\right)^{h}\left(y^{q}\right)^{h b^{\prime}}=\left(x^{h}\right)^{p}\left(y^{h b^{\prime}}\right)^{q} .
$$


By Theorem 7.5, there is an element $g$ that conjugates $x$ to $x^{g}$ and $y^{b}$ to $y^{g b^{\prime}}$. In particular, the signs $\iota\left(x, y^{b}\right)$ and $\iota\left(x^{g}, y^{g b^{\prime}}\right)$ coincide, so there is no cancellation. This concludes the proof.

\section{References}

[1] A Ángel, E Backelin, B Uribe, Hochschild cohomology and string topology of global quotient orbifolds, J. Topol. 5 (2012) 593-638 MR2971608

[2] A F Beardon, The geometry of discrete groups, Graduate Texts in Mathematics 91, Springer, New York (1983) MR698777

[3] K Behrend, G Ginot, B Noohi, P Xu, String topology for loop stacks, C. R. Math. Acad. Sci. Paris 344 (2007) 247-252 MR2292996

[4] M Boileau, S Maillot, J Porti, Three-dimensional orbifolds and their geometric structures, Panoramas et Synthèses 15, Soc. Math. France, Paris (2003) MR2060653

[5] J W Cannon, W J Floyd, R Kenyon, W R Parry, Hyperbolic geometry, from "Flavors of geometry" (S Levy, editor), Math. Sci. Res. Inst. Publ. 31, Cambridge Univ. Press (1997) 59-115 MR1491098

[6] M Chas, Combinatorial Lie bialgebras of curves on surfaces, Topology 43 (2004) 543-568 MR2041630

[7] M Chas, Minimal intersection of curves on surfaces, Geom. Dedicata 144 (2010) 25-60 MR2580416

[8] M Chas, F Krongold, An algebraic characterization of simple closed curves on surfaces with boundary, J. Topol. Anal. 2 (2010) 395-417 MR2718130

[9] M Freedman, J Hass, P Scott, Least area incompressible surfaces in 3-manifolds, Invent. Math. 71 (1983) 609-642 MR695910

[10] W M Goldman, Invariant functions on Lie groups and Hamiltonian flows of surface group representations, Invent. Math. 85 (1986) 263-302 MR846929

[11] L Greenberg, Finiteness theorems for Fuchsian and Kleinian groups, from "Discrete groups and automorphic functions" (W J Harvey, editor), Academic Press, London (1977) 199-257 MR0585138

[12] A Hatcher, Notes on basic 3-manifold theory, lecture notes (2000) Available at https://www . math. cornell. edu/ hatcher/3M/3Mdownloads.html

[13] M Kapovich, Hyperbolic manifolds and discrete groups, Progress in Mathematics 183, Birkhäuser, Boston (2001) MR1792613

[14] S Katok, Coding of closed geodesics after Gauss and Morse, Geom. Dedicata 63 (1996) 123-145 MR1413625 
[15] E Lupercio, B Uribe, M A Xicotencatl, Orbifold string topology, Geom. Topol. 12 (2008) 2203-2247 MR2431019

[16] J G Ratcliffe, Foundations of hyperbolic manifolds, 2nd edition, Graduate Texts in Mathematics 149, Springer, New York (2006) MR2249478

[17] P Scott, The symmetry of intersection numbers in group theory, Geom. Topol. 2 (1998) 11-29 MR1608688

[18] W P Thurston, The geometry and topology of three-manifolds, lecture notes, Princeton University (1979) Available at http://msri.org/publications/books/gt3m

Department of Mathematics, Stony Brook University

Stony Brook, NY 11794, United States

Department of Mathematics, Indian Institute of Science

Bangalore 560012, India

moira.chas@stonybrook.edu, gadgil@math.iisc.ernet.in

http://www.math.stonybrook.edu/ moira/,

http://math.iisc.ernet.in/ gadgil/

Received: 31 March 2015 Revised: 7 January 2016 